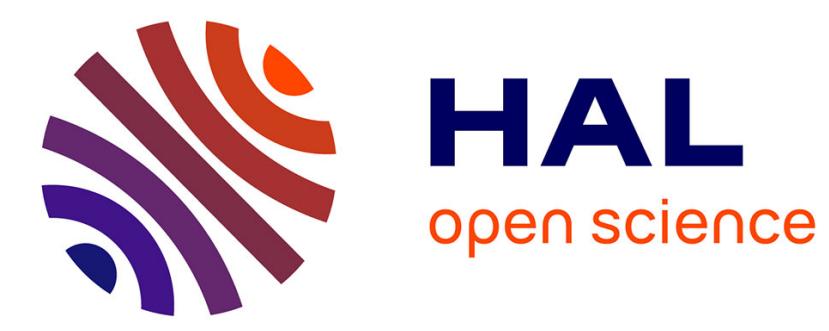

\title{
Aerosols in Healthy and Emphysematous In Silico Pulmonary Acinar Rat Models
}

Jessica M. Oakes, Philipp Hofemeier, Irene Vignon-Clementel, Josué Sznitman

\section{To cite this version:}

Jessica M. Oakes, Philipp Hofemeier, Irene Vignon-Clementel, Josué Sznitman. Aerosols in Healthy and Emphysematous In Silico Pulmonary Acinar Rat Models. Journal of Biomechanics, 2016, 49 (11), pp.2213-2220. 10.1016/j.jbiomech.2015.11.026 . hal-01244458

\section{HAL Id: hal-01244458 \\ https://hal.inria.fr/hal-01244458}

Submitted on 15 Dec 2015

HAL is a multi-disciplinary open access archive for the deposit and dissemination of scientific research documents, whether they are published or not. The documents may come from teaching and research institutions in France or abroad, or from public or private research centers.
L'archive ouverte pluridisciplinaire HAL, est destinée au dépôt et à la diffusion de documents scientifiques de niveau recherche, publiés ou non, émanant des établissements d'enseignement et de recherche français ou étrangers, des laboratoires publics ou privés. 


\section{Aerosols in Healthy and Emphysematous In Silico Pulmonary Acinar Rat Models}

Jessica M. Oakes ${ }^{1,2,3, *}$, Philipp Hofemeier ${ }^{4, *}$, Irene E. Vignon-Clementel ${ }^{2,3}$, Josué Sznitman ${ }^{4, * *}$

Keywords: computational fluid dynamics (CFD), emphysema, particle deposition, disease

modelling, alveoli

\footnotetext{
${ }^{*}$ Authors contributed equally

${ }^{* *}$ Corresponding Author

${ }^{1}$ Department of Mechanical Engineering, University of California Berkeley, Berkeley, CA 94709,USA

${ }^{2}$ INRIA Paris-Rocquencourt, 78153 Le Chesnay Cedex, France

${ }^{3}$ Sorbonne Universités, UPMC Univ Paris 6, Laboratoire Jacques-Louis Lions, 75252 Paris, France

${ }^{4}$ Department of Biomedical Engineering, Technion - Israel Institute of Technology, Haifa 32000, Israel
} 


\section{Abstract}

There has been relatively little attention given on predicting particle deposition in the respiratory zone of the diseased lungs despite the high prevalence of chronic obstructive pulmonary disease (COPD). Increased alveolar volume and deterioration of alveolar septum, characteristic of emphysema, may alter the amount and location of particle deposition compared to healthy lungs, which is particularly important for toxic or therapeutic aerosols. In an attempt to shed new light on aerosol transport and deposition in emphysematous lungs, we performed numerical simulations in models of healthy and emphysematous acini motivated by recent experimental lobar-level data in rats [17]. Compared to healthy acinar structures, models of emphysematous subacini were created by removing inter-septal alveolar walls and enhancing the alveolar volume in either a homogeneous or heterogeneous fashion. Flow waveforms and particle properties were implemented to match the experimental data. The occurrence of flow separation and recirculation within alveolar cavities was found in proximal generations of the healthy zones, in contrast to the radial-like airflows observed in the diseased regions. In agreement with experimental data, simulations point to particle deposition concentrations that are more heterogeneously distributed in the diseased models compared with the healthy one. Yet, simulations predicted less deposition in the emphysematous models in contrast to some experimental studies, a likely consequence due to the shallower penetration depths and modified flow topologies in disease compared to health. These spatial-temporal particle transport simulations provide new insight on deposition in the emphysematous acini and shed light on experimental observations.

\section{Introduction}

While computational models that describe the behaviour of inhaled particles in the respiratory acinar regions of the healthy lung have attracted broad attention [8, 10, 13,-15], little focus has yet been made on modelling the transport of aerosols in the diseased pulmonary acinus. To the 
best of our knowledge, no 3D in silico acinar models have attempted to address the fate of inhaled micron-sized aerosols in the context of pulmonary conditions such as emphysema. Emphysema is a progressively severe heterogeneous obstructive disease caused by inhalation of toxic gases and particles over a long period of time [11]. The disease is characterized by alveolar airspace enlargement caused by deterioration of the pulmonary tissue leading to a loss of interalveolar septa [30]. At its earliest stages the diseased lesions are heterogeneously distributed in the lung; however, as the disease progresses inflammation, protease activity, and remodelling leads to a more severe and widespread distribution of damaged tissue [24, 25]. Due to the increased resistance of the small airways and tissue compliance, the lung takes a longer time to empty [11], which may lead to ventilation asymmetry [19], air trapping [12], and ventilation deficiency [4]. As aerosol medications are increasingly used to either treat pulmonary or systemic diseases, it is imperative to understand deposition in both healthy and diseased lungs. While effective treatment of emphysema is still unavailable, recent animal studies have suggested that biphosphonate (alendronate) inhalation, commonly used to treat osteoporosis, may have therapeutic potential by blunting the inflammatory response of alveolar macrophages [31].

Previous in vivo [3, 17, 26] and in vitro studies [1, 18] have attempted to uncover the behaviour of inhaled particles in the emphysematous lung. However, likely due to the progressive nature of the disease, there remains a lack of agreement on whether there are more or less particles depositing in the emphysematous lung compared to a healthy one. For example, Oakes et al. [17] found enhanced deposition in elastase-treated rat lungs compared to healthy ones measured with Magnetic Resonance Imaging (MRI), in contrast to earlier measurements obtained in elastase-treated hamsters where a decreased deposition was measured [26]. Yet, both animal studies [17, 26] agreed on the enhanced heterogeneity in the distribution of aerosol deposition patterns in the diseased lungs. In a 3D scaled-up in vitro studies, Oakes et al. [18] and Berg et al. [1] determined a decrease in penetration depth in an emphysematous alveolar sac and acinar model compared to healthy ones and 
hypothesized that this would result in a decrease in deposition in the diseased models. This finding agrees with deceased deposition in emphysema, compared to healthy lungs, found in a stochastic model of various types of emphysema [23].

The advantage of numerically modelling the lung lies in the ability to investigate particle transport and deposition at temporal and spatial resolutions that are currently beyond reach with current state-of-the-art imaging modalities. Motivated by such shortcomings and available aerosol deposition data in rats [17], a computational framework has been recently developed to model airflow and particle transport in anatomically-reconstructed conducting airways of rats [20]. While deposition predictions between in vivo and in silico agreed well in healthy rats, similar agreement was not found for the emphysematous animals [19]. As this in silico model did not include the small airways and acinar region of the lung, the behaviour of particles once they reach the distal regions of the lung remains widely unknown. It is hypothesized that the enlarged airspaces and deterioration of the alveolar septa, characteristic of emphysema, will lead to noticeable differences in total and spatial distribution patterns of particles.

The main aims of this study were to numerically investigate the deposition patterns in healthy and diseased acini and to shed light on the transport mechanisms behind the enhanced deposition in emphysema found experimentally [19]. For such purpose, we adapted a numerical acinar framework recently developed [10] and compared deposition predictions between a healthy rat acinar model and two emphysematous cases. The emphysema models were created by enlarging airspaces and removing connecting alveolar septa in either a homogeneous or heterogeneous fashion. To facilitate comparison between our predictions and experimental data, both the ventilation (i.e. breathing patterns) and particle properties were chosen to match the conditions implemented in Oakes et al. [17]. By assessing the differences between healthy and diseased acini, our efforts aim to advance the knowledge of inhaled particles in the deep regions of the diseased lung and pinpoint the mechanisms responsible for the deposition differences between the healthy and emphysematous 
rats.

\section{Methods}

\section{Rat Acinar Geometry}

Three distinct multi-generational rat acinar domains were designed following a space-filling model of 3D polyhedral units [7, 27]. A healthy $(H)$, heterogeneous emphysematous $\left(E_{H e t}\right)$ and homogeneous emphysematous $\left(E_{\text {Hom }}\right)$ models were created (Fig. 1 1a-c), where each acinar network consists of up to six airway generations with a maximum of 277 polyhedral alveoli (Table 1). The resulting sub-acini capture sufficiently well realistic full acinar structures [13]. A healthy human acinar model [10] was scaled down by $15 \%$ to match dimensions of a rat acinus [21] at functional residual capacity $(F R C)$ since interspecies differences are overall minor (see limitations below for further discussion). The outer airway sleeve diameter, including the ducts and surrounding alveoli, was held constant at $86 \mu \mathrm{m}$ with a characteristic alveolar diameter of $35 \mu \mathrm{m}$. Airway ducts spanned a length of 56 to $85 \mu \mathrm{m}$, depending on generation.

In order to capture and integrate some of the emphysema-like morphological changes, the $H$ model was modified according to two characteristics features: (i) removing the inter-alveolar septal walls as highlighted in Fig. 1e (inset) and (ii) increasing the acinar volume of the model by adding additional polyhedral structures in the bifurcation regions (see Table 11). Thus, diseased regions were characterized as enlarged continuous airspaces without distinct alveolar cavities, in contrast to the normal regions (compare Fig. 1 1 a to d). The entire $E_{\text {hom }}$ model was defined as diseased and thus the emphysema-like changes were distributed throughout the model (see Fig. $1 \mathrm{~d}$ and Table 1). The $E_{\text {het }}$ model represents a non-uniform distribution of emphysema where two zones were created; a normal zone $(N)$ and a diseased zone $(D)$. The bottom right portion of the model was prescribed as diseased as highlighted in grey in Fig. 1 $1 \mathrm{~b}$ leaving the rest of the model as normal (Fig. 17). FRC values for each model, including the two regions of the $E_{\text {het }}$ model, are presented in Table 1, showing that FRC increases with emphysema severity. In order to underline the loss of 
septal walls, the number of alveolar cavities as well as the surface-to-volume ratio $S / V$ are shown in Table 1 ; here, we find that $S / V$ is approximately decreased by half for the $E_{\text {hom }}$ model compared to the healthy condition. Corresponding videos presenting the acinar models and their respective breathing motions are supplied in the Supplementary Material (SM).

\section{Respiration Curves}

A self-similar breathing motion was prescribed across the entire acinar domain to simulate cyclic expansion and contraction motion following previous works [9, 10, 27]. Realistic respiration curves, derived from rat ventilation studies [17, 20], were scaled for each of the acinar models in order to match realistic tidal volumes. Specifically, the time-dependent acinar volumes were defined as $V_{H, A}(t)=\alpha V_{H, T}(t)$ and $V_{E, A}(t)=\alpha V_{E, T}(t)$ for the $H$ and $E_{\text {hom }}$ models, respectively; note that the indices $A$ and $T$ indicate acinar and total lung, respectively. Assuming that the acinar volume fraction of the $H$ and $E_{h o m}$ models are identical, $\alpha$ was set to $F R C_{H, A} / F R C_{H, T}$, with $F R C_{H, T}=4.77 \mathrm{~mL}[22]$.

It is important to note that a straightforward scaling of the time-dependent volume curve is not feasible for the $E_{\text {het }}$ model as the tissue mechanics of the normal and diseased zones are different. Following a recent approach [19], we scaled the curves separately for each region based on a lumped model where respiratory resistance $(R)$ and compliance $(C)$ are in series. Assuming that the normal region of the lung correlates with the healthy rat lung, $R_{N}$ and $C_{N}$ were set to $R_{N}=R_{H, T} / \alpha_{N}$ and $C_{N}=C_{H, T} * \alpha_{N}$, where $R_{H, T}=0.098 \mathrm{~cm} \mathrm{H}_{2} \mathrm{O}-\mathrm{s}-\mathrm{cm}^{-3}$ and $C_{H, T}=0.236 \mathrm{~cm}^{3}\left(\mathrm{~cm} \mathrm{H}_{2} \mathrm{O}\right)^{-1}$. Here, $\alpha_{N}=\alpha F R C_{N, A} / F R C_{H, A}$ [19]. The respiratory volume curve of the normal region $\left(V_{N, A}(t)\right)$ was found by directly solving

$$
R_{N} \frac{d V_{N, A}(t)}{d t}+\frac{V_{N, A}(t)}{C_{N}}=P(t)-P_{p e e p}
$$

where $P(t)$ is the pressure measured at the trachea of the emphysematous rat during ventilation and $P_{\text {peep }}$ is the positive expiratory pressure of $1 \mathrm{~cm} \mathrm{H}_{2} \mathrm{O}$ [19]. The respiratory volume curve of the 
diseased region $\left(V_{D, A}(\mathrm{t})\right)$ was calculated as $V_{D, A}(t)=T V_{D, A} V_{E, T}(t) / T V_{E, T}$, where the tidal volume of the diseased region is defined as $T V_{D, A}=T V_{E, A}-T V_{N, A}$. The corresponding $V_{N, A}$ and $V_{D, A}$ were prescribed to the normal and diseased regions of the $E_{\text {het }}$ model in the 3D flow simulations.

The resulting volume curves (i.e. $V_{A}(t)$ normalized by $F R C_{A}$ ) and flow rates over the cycles are shown for each acinar model in Fig. $2 \mathrm{a}$ and $\mathrm{b}$, respectively. Note that the tidal volumes were the same for each model and were slightly larger in the diseased region compared to the normal region of the $E_{\text {het }}$ model (Table 1 and Fig. 2). As shown in Oakes et al. [19], the decay rates of $V_{E, A}$ and $V_{D, A}$ were slower compared to the corresponding healthy curves (Fig. 2 a). This resulted in lower peak flow rates during exhalation (Fig. 2p). Flow rates during inspiration were nearly the same for the $H$ and $E_{\text {hom }}$ models because all the rats were ventilated with identical settings [20]. The diseased zone of $E_{\text {het }}$ finished filling slightly after the corresponding normal zone, due to the longer time constant of the diseased region as shown in the inset of Fig. $2 \mathrm{~b}$, namely $\mathcal{T}=R C$ such that $\mathcal{T}_{N, A}=0.023 \mathrm{~s}$ and $\mathcal{T}_{D, A}=0.045 \mathrm{~s}$.

\section{Flow and Particle Simulations}

Airflow and particle transport simulations were performed in OpenFOAM (Open Source Field Operation and Manipulation, Version 2.1.1). Airflow was modeled as a continuum using the finite volume method (FVM), where the Navier-Stokes equations were solved on an arbitrary Lagrangian-Eulerian (ALE) framework assuming air to be incompressible, Newtonian and at constant temperature $\left(37^{\circ} \mathrm{C}\right)$. Further details on the numerical solver and discretization models has been recently discussed [10]. Briefly, airflow motion was induced by the expansion (inhalation) and contraction (exhalation) of the domain as described in the section above. At the inlet/outlet a constant pressure was imposed, since the flow field is generated as a result of the prescribed domain motion; note that the absolute pressure is not needed to solve the transport equation. Following previous convergence studies, a total of $1.4 \mathrm{M}$ tetrahedral cells $(H$ model) was used to discretize the acinar domain [10], where a total of four different mesh sizes were analyzed ranging from $0.7 \mathrm{M}$ to 
$5 \mathrm{M}$ cells. Here, a final mesh size of $1.4 \mathrm{M}$ tetrahedral cells was found to faithfully capture velocities in the $H$ model, which is anticipated to experience the highest velocity gradients compared to the two other models. A dynamic time stepping was used to maintain the Courant-Friedrichs-Lewy condition $(C F L<1)$ to capture rapid changes in the flow (i.e. velocity gradients) during exhalation [10]. The developed numerical algorithm was compared and validated with experimental measurements [5] as well as analytical solutions [9, 10].

Neglecting electrostatic and hygroscopic effects, it is widely acknowledged that the main forces acting on airborne spherical and inert particles at the micron scale are viscous drag (convection), gravitational sedimentation, and Brownian diffusion [28]. Using Lagrangian particle tracking methods, aerosol kinematics were solved from the particle momentum equation accounting for drag, gravity and stochastic diffusion [10], where a one-way fluid particle coupling was used since low particle concentrations are anticipated in the most distal acinar generations [10]. Particles were injected by seeding particles (diameter $=1 \mu \mathrm{m}$, density $=1 \mathrm{~g} / \mathrm{cm}^{3}$ ) according to [17] continuously over the first inspiration as a function of the local (and unsteady) velocity, thus mimicking a constant concentration of injected particles. A total of 170,000 particles were injected and tracked over two breathing cycles. Only $\sim 1 \%$ of the injected particles remained airborne in the model after the second breathing cycle.

\section{Results}

To assess the nature of acinar flow structures under emphysematous conditions, flow streamlines for the $E_{\text {het }}$ model are shown in Fig. 3 at peak inspiration $(t=\tau / 8)$, where flow patterns for the healthy and diseased regions are simultaneously compared. Flow topologies in ducts and alveoli of healthy acini show characteristic configurations that evolve as a function of acinar generation depth. Indeed, in proximal generations alveolar flows separate as a result of relatively high-shear flows in the duct compared to slow, recirculating flows in the alveolar cavity (Fig. 3 , top left). As acknowledged to exist in distal acinar generations [6, 28], streamlines within alveolar cavities 
feature radial-like structures, thus following more closely the motion of the alveolar walls in the absence of strong ductal flows (Fig. 3, top right). Alveolar flow patterns transition to half-open streamlines in medial generations (Fig. 3, bottom left), underlining the coupling between ductal shear flows and alveolar wall motion. In contrast to healthy regions, due to the absence of septa walls, flow patterns in the diseased regions lack the characteristic separation between ductal and alveolar flow regims. Instead, slow, quasi-parallel streamlines form across the ductal segment, a feature previously seen with in vitro models of terminal sacs [18].

Instantaneous particle positions are shown in Fig. 4 at three characteristic time points: end of first inhalation $(\mathrm{a}, \mathrm{d}, \mathrm{g})$, end of second inhalation $(\mathrm{b}, \mathrm{e}, \mathrm{h})$ and at the end of the second breath $(\mathrm{c}, \mathrm{f}, \mathrm{i})$, where the color-coding indicates airborne (red) or deposited (blue) particles. The majority of particles remain airborne after the first inhalation $(t=0.5 \tau$, Fig. $4 \mathrm{a}, \mathrm{d}$ and $\mathrm{g})$ and do not penetrate as far in the emphysematous regions compared to the normal ones. In particular, particles in the $H$ model (Fig. 4a) are carried with the ductal flow deep into the acinar structure, whereas the lack of ductal structures in the emphysematous regions (e.g. $E_{\text {hom }}$, Fig. $4 \mathrm{~g}$ ) causes the velocities to slow as the cross-sectional area increases. Additionally, most particles either deposited or were exhaled by the end of the second inhalation in the $H$ model, in contrast to the $E_{\text {hom }}$ and $E_{\text {het }}$ models, where particles remained airborne in the diseased regions. However, most particles deposit before the second exhalation ( $t=1.5 \tau$, Fig. $4 \mathrm{~b}, \mathrm{~h}$ and e). In contrast, the majority of particles either deposit or have exited the domain at the proximal inlet/outlet of the $H$ and $E_{\text {het }}$ models, respectively. At the end of the second breath (Fig. 4t,f and i), a small fraction of particles remain airborne in the emphysematous regions. Videos illustrating the dynamic behavior of inhaled particles are provided for each model (see SM).

Quantitatively, total deposition is larger in the $H$ model (49\%) compared to the diseased models ( $E_{\text {het }}: 38 \%, E_{\text {hom }}: 18 \%$ ), see Fig. 5. While the majority of deposition occurs during the first breath, aerosols continue to deposit throughout the respiration cycles (Fig. 5h). The rate of particle 
deposition is highest at the start of exhalation (Fig. 5), when flow rates are highest (Fig. 2b). We note that the deposition fraction in the $H$ model plateaus throughout the second breathing cycle, whereas $E_{h e t}$ and $E_{h o m}$ exhibit a small incline, due to a decreased deposition rate in the emphysematous regions. In an attempt to capture physiologically-relevant deposition metrics, regional deposition data are extracted and distinguished according to (i) alveolar (i.e. deposition in healthy alveolar cavities), (ii) ductal (i.e. deposition on the alveolar ring openings and the connecting elements between the generations), and (iii) diseased regions (i.e. deposition in regions where the ductal and alveolar structures are degenerated). Here, we find that the majority of particles deposit in the ductal regions of the $H$ and $E_{\text {het }}$ models (Fig.5p); only $14 \%$ of the particles deposit inside the alveolar cavities of the $H$ model. The majority of deposition is seen in the normal regions (normal: 87\%, diseased: $13 \%$ ) for the $E_{\text {het }}$ model. As the diseased regions of the $E_{h o m}$ and $E_{h e t}$ models do not contain septa or distinct alveolar cavities (Fig. 11, inset), deposition could not be discriminated between ductal and alveolar regions. Accordingly, particles that deposit in the diseased regions of the $E_{\text {het }}$ and $E_{\text {hom }}$ models are labeled as such (Fig. 5, b).

Additionally, we assessed final particle deposition penetration depths according to centerline distances (qualitatively shown in Fig. 4, right column). Here, the centerline starts at the entrance of the acinar model, follows the duct and ends at the particle deposition site (Fig. 6). It should be underlined that the penetration depth measured is not equivalent to the particle pathline. Particles penetrated the deepest in the $H$ model with a mean of $l_{H}=0.23 \mathrm{~mm}$ compared to the emphysematous ones with $l_{E_{h e t}}=0.2 \mathrm{~mm}$ and $l_{E_{h o m}}=0.16 \mathrm{~mm}$, respectively. We statistically compared these mean values using a non-parametric Wilcoxon signed rank test with Bonferroni correction (R programming language, Version 3.2.0). Cross-testing all models, we find that means are significantly different between all models $(p \ll 0.001)$. Qualitatively, particle deposition fractions are similar between the $H$ and $E_{\text {het }}$ models for particles that deposited within $0.2 \mathrm{~mm}$ of the entrance. More particles deposited in the $H$ model compared to the $E_{\text {het }}$ model for penetration depths $>0.2 \mathrm{~mm}$. 
The drop in particle deposition in the distal generations of the $E_{\text {het }}$ region is correlated with the entrance to the diseased region ( $0.24 \mathrm{~mm}$ from the entrance). Indeed, deposition mainly occurs at the entrance and first generations of the $E_{\text {hom }}$ model while distal regions are nearly depleted of particles (Figs. 4 and 6).

Finally, we assessed particle concentrations by superimposing a 3D voxel grid and counting the number of deposited particles per voxel (Fig. 7). Here, the sensitivity of concentration with respect to the voxel size was first tested (i.e. the larger the voxel size the more particles contained within the voxel); as the trends were independent of voxel size, a final voxel size of $10 \mu \mathrm{m}$ lateral length (approximately $1 / 3$ of the alveolar diameter) was eventually chosen. Generally, particle concentration was highest at the entrance of $H$ (Fig. 7a) and $E_{\text {het }}$ models (Fig. 7p). Furthermore, concentration is relatively uniform in the $H$ model as the particles reached all generations of the model. In contrast, fewer particles reached, and thus deposited in the diseased regions of the $E_{\text {het }}$ and $E_{\text {hom }}$ models. Motivated by the deposition data collected in rats [17], the relative dispersion $(R D)$ was calculated by dividing the standard deviation of all voxel concentrations by their average. A clear trend is noted where $R D$ increases with increasing emphysematous region, such that $E_{h o m}$ yields the largest $R D$ (Fig. $7 \mathrm{~d}$ ). This finding further underlines localized deposition phenomena in emphysematous regions in $E_{\text {het }}$ and $E_{\text {hom }}$, as noted in Fig.7b,c and previously in Fig. 6.

\section{Discussion}

Despite the high prevalence of emphysema, there are few studies aimed at understanding differences in particle deposition between healthy and emphysematous lungs [3, 17, 26]. Due to experimental feasibility, these studies only report global deposition, thus rendering it unclear which mechanisms are the underlying causes of deposition differences in emphysema. While it has been previously shown that an increase in tissue compliance results in enhanced delivery of airborne particles to the diseased lung regions [19], the fate of particles once they reach the alveolated airways remains largely unknown. Motivated by recent experimental [17] and numerical studies [19, 20] 
in rat lungs, we performed particle-laden airflow simulations in multi-generational acinar models (Fig. 1). One healthy and two distinct emphysema models were designed in an effort to assess disease severity, where diseased regions were distributed either heterogeneously ( $E_{\text {het }}$, Fig. 11) or homogeneously ( $E_{\text {hom }}$, Fig. 11d). Emphysematous regions were created by removing the septa between alveolar cavities and enlarging the alveolar volume. As such, the emphysematous models represent panacinar emphysema [29, 30], a type of emphysema associated with alpha1-antitrypsin deficiency found in elderly patients [29]. Panacinar emphysema is analogous to the one elastase creates in rat lungs [2, 16].

Our findings indicate that micron-sized particles deposit mainly during the first breathing cycle and the deposition rate is maximum at the start of the first exhalation (Fig. 51). Compared to the $E_{\text {hom }}$ and $E_{\text {het }}$ models, deposition is enhanced in the healthy $(H)$ model (Fig. 5) due to the presence of inter-septal walls and alveolar rings (Fig.11) and the larger normalized tidal volume in the $H$ model (Fig. 2) compared to the emphysematous models. Absolute tidal volumes of all models were kept the same to match the experimental ventilation settings [17], such that deposition differences between the models are anticipated to result from geometric differences and ensuing flow characteristics. Namely, inter-septal walls under healthy conditions create flow boundaries between the alveolar cavities and the acinar ducts [6, 9, 28], resulting in relatively fast ductal flows compared to slow, recirculating flows in the alveolar cavities (Fig. 3, proximal insert). Consequently, the particle-laden air is carried deeper into the acinus of the $H$ model, resulting in enhanced penetration depths in health compared to emphysema (see Fig. 4 and SM), as was previously shown in an in vitro alveolar sac [18] and acinar model [1]. This resulted in fewer particles depositing in the distal areas of the models (Fig. 4 and Fig. 6).

Total deposition was found to be higher in the $E_{h e t}$ model compared to the $E_{h o m}$ model with the majority of particles depositing in the normal regions (Fig 5), despite nearly the same regional FRC (Table 1). Comparing the $H$ and $E_{\text {het }}$ model, slightly more particles deposited on the healthy 
airways leading to the diseased region of the $E_{\text {het }}$ model caused by larger tidal volume of the distal diseased segments and accordingly, resulted in more particle-laden air to travel through these airways. Particle deposition was higher in the outer sleeve (i.e. the former alveolar cavities) of the emphysematous models compared to the alveolar cavities in the $H$ model, as the $E_{\text {hom }}$ and $E_{\text {het }}$ models hold no inter-septal walls. Particles that entered the emphysematous regions were more likely to remain suspended after the first breath (Fig. 4) and travelled slightly deeper during the subsequent breath in the diseased zones (see Fig. 4 and SM). This is a result of a net gravitational sedimentation with a deviation from the original pathline such that particles are not exhaled. While airborne particles may deposit upon subsequent breaths, only $\sim 1.2 \%$ of inspired particles remained suspended representing a minor role on total deposition. Particles may become trapped if the small airways collapse upon exhalation, a phenomenon not captured here, but known to occur in emphysema [12]. This will likely result in enhanced particle deposition in these regions given that particles will have more time to migrate to the airway walls. Such an effect could explain the disparities between model predictions and the experimental work by Oakes et al. [17], where higher deposition was measured in disease.

While direct comparison of model predictions with in vivo experimental data is not currently feasible, as a single voxel of the experimental MRI data contains a combination of small airways and acinar structures, some general comparisons may be made. First, in contrast to the rat experiments [17], we predicted less deposition in emphysema; this latter trend is however in agreement with an in vivo study performed in hamsters [26], an in vitro model [18] and a stochastic model [23]. Here, the relative dispersion $(R D)$ represents a measure of the uniformity of the deposited particle concentration, where a larger $R D$ thus indicates a more heterogeneous distribution of deposited particles. In agreement with the experimental data of Oakes et al. [17], we showed that $R D$ was larger in the emphysematous models compared to the $H$ models (Fig.7 $7 \mathrm{~d}$ ). Namely, the $E_{\text {hom }}$ model had the largest $R D$ compared to the other two models (Fig. $7 \mathrm{~d}$ ), underlining regions of high particle 
concentration (hot spots) and areas where particles did not deposit (Fig. 7k).

In a recent numerical study in the rat conducting airways, Oakes et al. [19] predicted that, due to the enhanced respiratory compliance, more particle-laden air would reach the respiratory zone of the diseased regions of a heterogenous emphysematous lung compared to normal regions. However, the fate of the particles once they enter the respiratory zone, and thus the influence of the morphometric changes that occur in emphysema was not investigated. This resulted in a non-favourable comparison between the numerical predictions and the experimental data for the emphysematous rats. Indeed, Oakes et al. [17] showed that particle deposition was higher in the healthy lobes of the emphysematous rat compared to the diseased lobes. Motivated by the inability of the previous numerical study [19] to uncover the mechanisms behind the deposition differences between the healthy and emphysematous rats, we have shown that in the absence of alveolar rings in the diseased regions of the $E_{\text {het }}$ model (Fig. 1), more particles deposit in the normal regions of the $E_{\text {het }}$ model compared to the diseased regions. Hence, a heterogeneous distribution of emphysema leads to higher deposition efficiency in the normal regions of the lung compared to the diseased ones; this latter finding may help explain the enhanced deposition found experimentally in the normal regions of the rat [17].

Characterizing an inherently heterogeneous disease such as emphysema is challenging and entails a set of reasonable assumptions and limitations. The numerical limitations of the utilized solver, boundary conditions and computational mesh were recently discussed [10]. Instead, we focus on discussing limitations pertaining to modelling emphysema. To transform the human subacinar model [10] to dimensions representative of a rat, a uniform scaling was assumed between rats and humans, and thus the smaller ratio of duct length to duct diameter ratio in rats [21] compared to humans [33] was not accounted for. However, as the goal of this study was to compare the influence of homogeneously and heterogeneously distributed emphysema on particle deposition, we anticipate that this choice of scaling will have little influence on the results. As mentioned, 
airway collapse is common during emphysema, a feature not mimicked in the present acinar model. Airway collapse may raise deposition fractions in the diseased regions. While deterioration of alveolar septa and enhanced alveolar volume are characteristic of emphysema, our model does not capture all changes that occur with emphysema. For example, alveoli may increase in size and change in shape [29], potentially further decreasing deposition in disease. Due to computational costs, it is currently infeasible to model the entire lung for all spatial and temporal dimensions, thus direct comparison between model predictions and experimental data cannot be made. The present work would certainly benefit from ventilation distribution maps [4, 12] or further experimental data in deposition in the lungs [32]. While this study addresses rat lungs, changes in flow structures in human lungs between the normal and diseased regions are likely to bear resemblance, where similar results could be anticipated.

By simulating particle transport in healthy and emphysematous acini, we were able to uncover the potential influence of emphysema-like geometric changes on acinar deposition. By implementing similar flow curves and particle properties of recent experiments in rat lungs [17, 20], comparison between our numerical predictions and experimental data were made. Our main findings are decreased deposition in the emphysematous models compared to the healthy model as well as increased dispersion in the diseased acini. Particle deposition in emphysematous acini is biased towards proximal acinar generations, while relative dispersion of particle concentrations is increased. As these findings are not entirely in agreement with previous studies (e.g. Oakes et al. [17]), other mechanisms are anticipated to influence deposition outcomes between the healthy and emphysematous rats. Hence, physiological factors such as small airway collapse, particle trapping and/or whole-lung heterogeneity, are hypothesized to be responsible for the enhanced deposition found experimentally. 


\section{Acknowledgements}

J. M. Oakes was supported by a Whitaker International Scholarship administrated by the International Institute of Education, an INRIA Associated Team Postdoctoral Grant and an University of California Presidential Postdoctoral Fellowship. P. Hofemeier and J. Sznitman were supported by the Israel Science Foundation (Grant nr. 990/12).

\section{Conflict of interest}

The authors have no conflict of interest related to the work presented in this manuscript.

\section{Supplementary Information}

SM1: 3D visualization of the $H$ (SM1a.avi), $E_{\text {het }}$ (SM1b.avi) and $E_{\text {hom }}$ model (SM1c.avi), where light grey indicates healthy and dark grey diseased regions. Rotation of the domain is first shown to illustrate the bifurcating acinar tree structure; next, a representative breathing cycle is shown highlighting the expansion and contraction of the domain. Note the asynchronous breathing pattern of the healthy and diseased zone in SM1b.

SM2: Particle positions over two complete breathing cycles for the $H$ (SM2a.avi), $E_{\text {het }}$ (SM2b.avi) and $E_{\text {hom }}$ model (SM2c.avi): Color-coding indicates airborne (red) and deposited (blue) particles.

\section{References}

[1] Berg, E.J., Robinson, R.J., 2011. Stereoscopic particle image velocimetry analysis of healthy and emphysemic alveolar sac models. Journal of Biomechanical Engineering 133, 061004. URL: http://www.ncbi.nlm.nih.gov/pubmed/21744924, doi:10.1115/1.4004251

[2] Borzone, G., Liberona, L., Olmos, P., Sáez, C., Meneses, M., Reyes, T., Moreno, R., Lisboa, C., 2007. Rat and hamster species differences in susceptibility to elastase-induced pulmonary emphysema relate to differences in elastase inhibitory capacity. American Journal of Physiology. Regulatory, Integrative and Comparative Physiology 293, R1342-9. 
[3] Brand, P., Schulte, M., Wencker, M., Herpich, C.H., Klein, G., Hanna, K., Meyer, T., 2009. Lung deposition of inhaled alpha1-proteinase inhibitor in cystic fibrosis and alpha1antitrypsin deficiency. The European Respiratory Journal 34, 354-60.

[4] Emami, K., Chia, E., Kadlecek, S., Macduffie-Woodburn, J.P., Zhu, J., Pickup, S., Blum, A., Ishii, M., Rizi, R.R., 2011. Regional correlation of emphysematous changes in lung function and structure: a comparison between pulmonary function testing and hyperpolarized MRI metrics. Journal of Applied Physiology 110, 225-235.

[5] Fishler, R., Hofemeier, P., Etzion, Y., Dubowski, Y., Sznitman, J., 2015. Particle dynamics and deposition in true-scale pulmonary acinar models. Scientific Reports .

[6] Fishler, R., Mulligan, M.K., Sznitman, J., 2013. Acinus-on-a-chip: A microfluidic platform for pulmonary acinar flows. Journal of Biomechanics 46, 2817-2823.

[7] Fung, Y.C., 1988. A model of the lung structure and its validation. Journal of Applied Physiology 64, 2132-2141.

[8] Harding, E.M., Robinson, R.J., 2010. Flow in a terminal alveolar sac model with expanding walls using computational fluid dynamics. Inhalation Toxicology 22, 669-78.

[9] Hofemeier, P., Sznitman, J., 2014. Role of alveolar topology on acinar flows and convective mixing. Journal of Biomechanical Engineering 136, 061007.

[10] Hofemeier, P., Sznitman, J., 2015. Revisiting acinar particle transport: convection, sedimentation, diffusion and their interplay. Journal of Applied Physiology 118, 1375-1385.

[11] Hogg, J.C., 2004. Pathophysiology of airflow limitation in chronic obstructive pulmonary disease. Lancet 364, 709-721. 
[12] Jacob, R.E., Carson, J.P., Thomas, M., Einstein, D.R., 2013. Dynamic multiscale boundary conditions for 4D CT of healthy and emphysematous rats. PloS One 8, e65874.

[13] Khajeh-Hosseini-Dalasm, N., Longest, P.W., 2015. Deposition of particles in the alveolar airways: Inhalation and breath-hold with pharmaceutical aerosols. Journal of Aerosol Science $79,15-30$.

[14] Kumar, H., Tawhai, M.H., Hoffman, E.a., Lin, C.L., 2009. The effects of geometry on airflow in the acinar region of the human lung. Journal of Biomechanics 42, 1635-42.

[15] Ma, B., Darquenne, C., 2011. Aerosol deposition characteristics in distal acinar airways under cyclic breathing conditions. Journal of Applied Physiology 110, 1271-1282.

[16] March, T.H., Green, F.H.Y., Hahn, F.F., Nikula, K.J., 2000. Animal models of emphysema and their relevance to studies of particle induced disease. Inhalation Toxicology 12, 155-187.

[17] Oakes, J.M., Breen, E., Scadeng, M., Tchantchou, G.S., Darquenne, C., 2014a. MRI-based measurements of aerosol deposition in the lung of healthy and elastase-treated rats. Journal of Applied Physiology 116, 1561-1568.

[18] Oakes, J.M., Day, S., Weinstein, S.J., Robinson, R.J., 2010. Flow field analysis in expanding healthy and emphysematous alveolar models using particle image velocimetry. Journal of Biomechanical Engineering 132, 021008-1-9.

[19] Oakes, J.M., Marsden, A.L., Grandmont, C., Darquenne, C., Vignon-Clementel, I.E., 2015. Distribution of aerosolized particles in healthy and emphysematous rat lungs: Comparison between experimental and numerical studies. Journal of Biomechanics 48, 1147-1157.

[20] Oakes, J.M., Marsden, A.L., Grandmont, C., Shadden, S.C., Darquenne, C., VignonClementel, I.E., 2014b. Airflow and particle deposition simulations in health and emphysema: from in vivo to in silico animal experiments. Annals of Biomedical Engineering 42, 899-914. 
[21] Rodriguez, M., Bur, S., Favre, A., Weibel, E.R., 1987. Pulmonary acinus: geometry and morphometry of the peripheral airway system in rat and rabbit. The American Journal of Anatomy 180, 143-55.

[22] Rubio, M.L., Sánchez-Cifuentes, M.V., Peces-Barba, G., Verbanck, S., Paiva, M., González Mangado, N., 1998. Intrapulmonary gas mixing in panacinar- and centriacinar-induced emphysema in rats. American Journal of Respiratory and Critical Care Medicine 157, 237-45.

[23] Sturm, R., Hofmann, W., 2004. Stochastic simulation of alveolar particle deposition in lungs affected by different types of emphysema. Journal of Aerosol Medicine 17, 357-72.

[24] Suki, B., Lutchen, K.R., Ingenito, E.P., 2003. On the progressive nature of emphysema: Roles of proteases, inflammation, and mechanical forces. American Journal of Respiratory and Critical Care Medicine 168, 516-521.

[25] Suki, B., Parameswaran, H., 2014. Computational modeling helps uncover mechanisms related to the progression of emphysema. Drug Discovery Today: Disease Models 70, 42454249.

[26] Sweeney, T.D., Brain, J.D., Leavitt, S.a., Godleski, J.J., 1987. Emphysema alters the deposition pattern of inhaled particles in hamsters. The American Journal of Pathology 128, $19-28$.

[27] Sznitman, J., 2009. Convective gas transport in the pulmonary acinus: Comparing roles of convective and diffusive lengths. Journal of Biomechanics 42, 789-792.

[28] Sznitman, J., 2013. Respiratory microflows in the pulmonary acinus. Journal of Biomechanics 46, 284-98.

[29] Takahashi, M., Fukuoka, J., Nitta, N., Takazakura, R., Nagatani, Y., Murakami, Y., Otani, 
H., Murata, K., 2008. Imaging of pulmonary emphysema: a pictorial review. International Journal of Chronic Obstructive Pulmonary Disease 3, 193-204.

[30] Thurlbeck, W.M., Muller, N.L., 1994. Emphysema: Definition, Imaging, and Quantification. American Journal of Roentgenology 163, 1017-1025.

[31] Ueno, M., Maeno, T., Nishimura, S., Ogata, F., Masubuchi, H., Hara, K., Yamaguchi, K., Aoki, F., Suga, T., Nagai, R., Kurabayashi, M., 2015. Alendronate inhalation ameliorates elastase-induced pulmonary emphysema in mice by induction of apoptosis of alveolar macrophages. Nature Communications 6, 6332-6345.

[32] Wang, H., Sebrié, C., Ruaud, J.P., Guillot, G., Bouazizi-Verdier, K., Willoquet, G., Maître, X., Darrasse, L., de Rochefort, L., 2015. Aerosol deposition in the lungs of spontaneously breathing rats using Gd-DOTA-based contrast agents and ultra-short echo time MRI at 1.5 Tesla. Magnetic Resonance in Medicine In Press.

[33] Weibel, E., 1965. Morphometry of the human lung, Springer. 


\section{List of Figures}

1 Schematic of the multi-generational acinar models of the 3D computational domain for the: (a) healthy, $H$, (c) heterogeneous emphysematous, $E_{H e t}$, and (d) homogeneous emphysematous $E_{\text {Hom }}$ cases. Light grey and dark grey denote the normal and diseased regions, respectively. A diagram of the acinar tree is shown in (b) where the diseased region of the $E_{H e t}$ model is highlighted in grey. Inset in (c) depicts the alveolar structure where inter-septal walls are outlined in purple (light grey region). No inter-septal walls are present in the diseased regions (dark grey

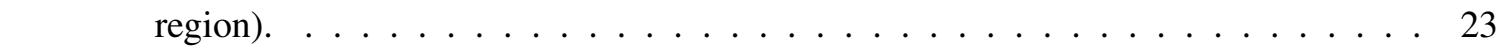

2 Inspired volume curves, $V_{A}(t)$, normalized by the $F R C_{A}$ of the three models (top . panel), and corresponding flow rates (bottom panel) for the three simulation cases. Volume (normalized by $F R C_{A}$ of the whole model) and corresponding flow curves for the diseased and normal zones are shown separately for the $E_{\text {het }}$ case. . . . . . . 24 3 Instantaneous 2D projections of representative 3D flow streamlines is shown at peak inspiration $(t=\tau / 8)$ for the $E_{\text {het }}$ model. Streamlines are color-coded (logarithmic scale) according to the local velocity magnitudes normalized by the maximal velocity at the inlet of the model. Four regions of the model are highlighted in the respective insets: proximal, medial, distal and diseased. . . . . . . . . . . . . 25

a 4 Snapshots of particle locations in the three acinar models captured at three time points during the breathing cycle. Particles in red are airborne (i.e. not deposited) and particles in blue are deposited. Corresponding movies of particle motions for the different cases are provided in the supplementary material (SM). . . . . . . . . 26

5 (a) Deposition fraction as a function of cumulative breathing cycles. Particles were injected throughout the inspiration phase of the first breath, from $t=0 \tau$ to $t=0.5 \tau$. (b) Final deposition fraction partitioned according to alveolar and ductal regions; the corresponding deposition fraction in the region of the emphysematous models is shown. Note that for such cases, the diseased regions cannot be distinguished according to alveolar and ductal regions in the absence of alveolar walls. . . . . . . 27 
473

474

475

476

477

478

479

480

481

482

483

484

485

Deposition fraction plotted as a function of the penetration depth measured from 1 the acinar entrance for the three acinar models, respectively. Penetration depths were taken after the second breath for deposited particles only, thus remaining airborne particles were not included in the calculation. Note that the penetration depths only assess the distance to the inlet of the domain, by calculating a particle's distance to the centreline of the duct and subsequently the distance (following the centreline of the duct) to the inlet. . . . . . . . . . . . . . . . . . 28

7 Deposited particle concentrations for the: (a) $H$, (b) $E_{\text {het }}$ and (c) $E_{\text {hom }}$ acinar models, respectively. Here, concentration is defined as the number of particles deposited within a voxel size with length of $10 \mu \mathrm{m}$. Panel (d) shows the relative dispersion $(R D)$, defined as the standard deviation normalized by the mean of the particle concentration for each acinar model [17]. Note that areas where particles did not deposit on were included in the $R D$ calculation. . . . . . . . . . . . . . . . 29 

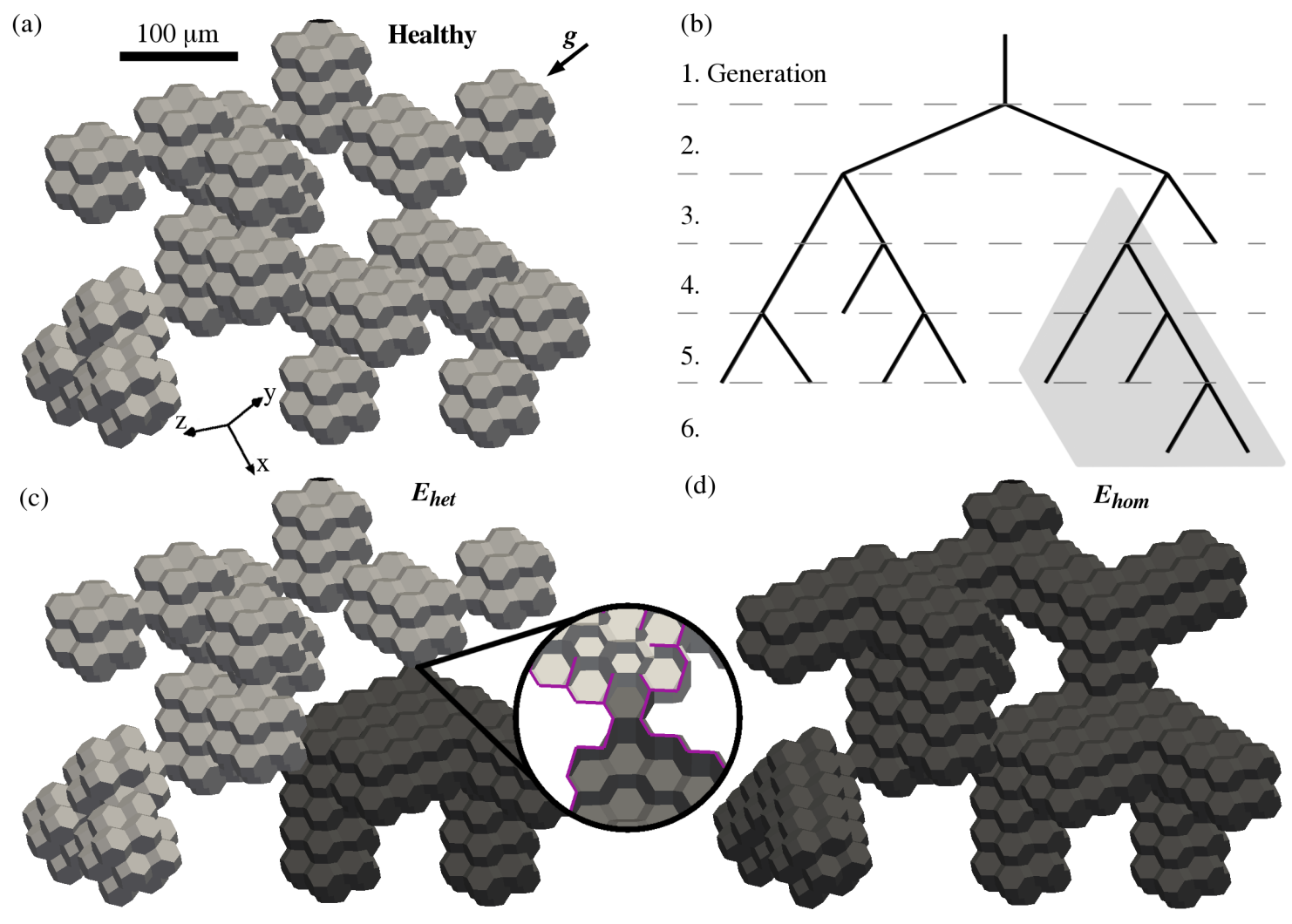

Figure 1: Schematic of the multi-generational acinar models of the 3D computational domain for the: (a) healthy, $H$, (c) heterogeneous emphysematous, $E_{H e t}$, and (d) homogeneous emphysematous $E_{H o m}$ cases. Light grey and dark grey denote the normal and diseased regions, respectively. A diagram of the acinar tree is shown in (b) where the diseased region of the $E_{H e t}$ model is highlighted in grey. Inset in (c) depicts the alveolar structure where inter-septal walls are outlined in purple (light grey region). No inter-septal walls are present in the diseased regions (dark grey region). 


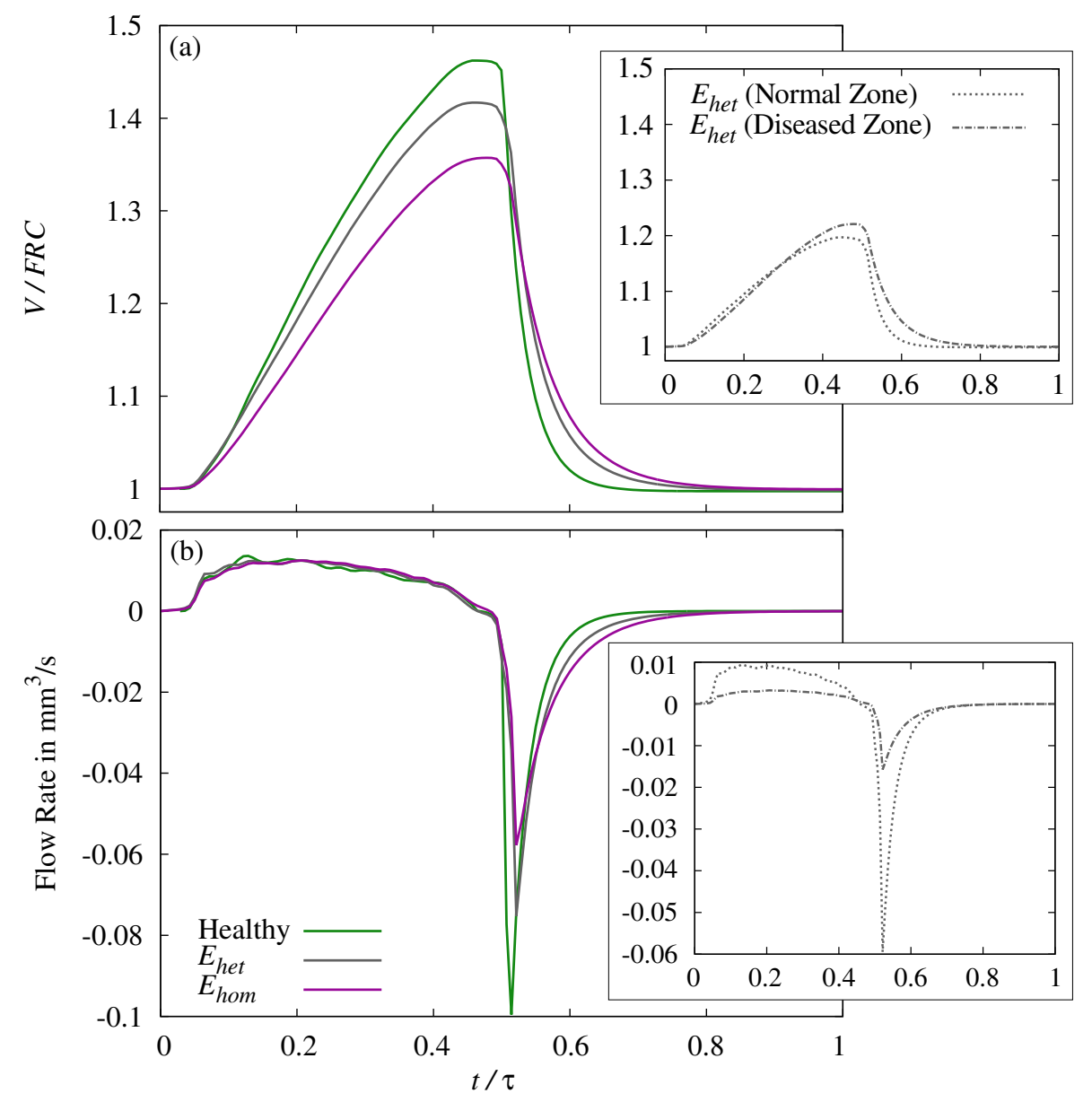

Figure 2: Inspired volume curves, $V_{A}(t)$, normalized by the $F R C_{A}$ of the three models (top panel), and corresponding flow rates (bottom panel) for the three simulation cases. Volume (normalized by $F R C_{A}$ of the whole model) and corresponding flow curves for the diseased and normal zones are shown separately for the $E_{\text {het }}$ case. 


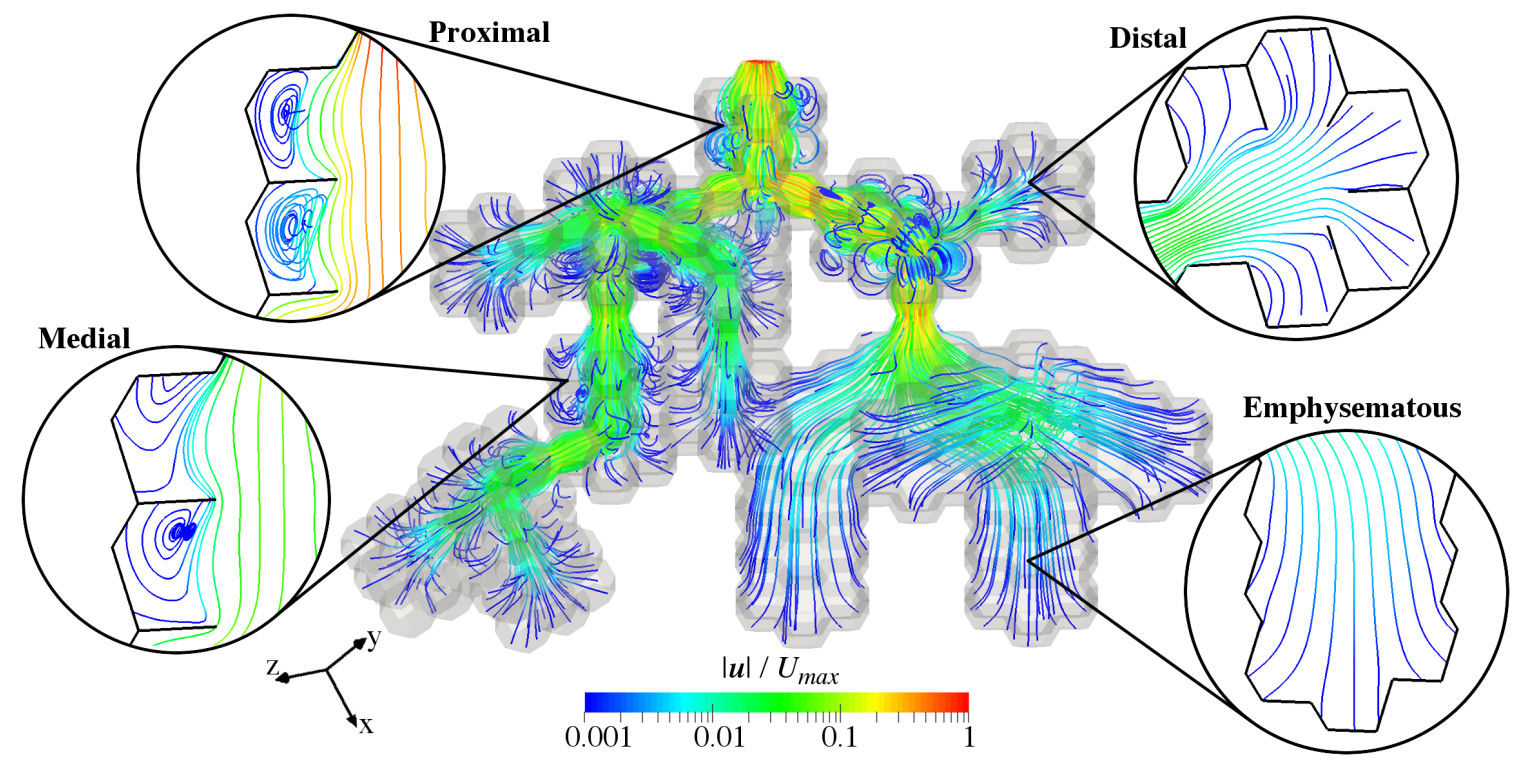

Figure 3: Instantaneous 2D projections of representative 3D flow streamlines is shown at peak inspiration $(t=\tau / 8)$ for the $E_{\text {het }}$ model. Streamlines are color-coded (logarithmic scale) according to the local velocity magnitudes normalized by the maximal velocity at the inlet of the model. Four regions of the model are highlighted in the respective insets: proximal, medial, distal and diseased. 

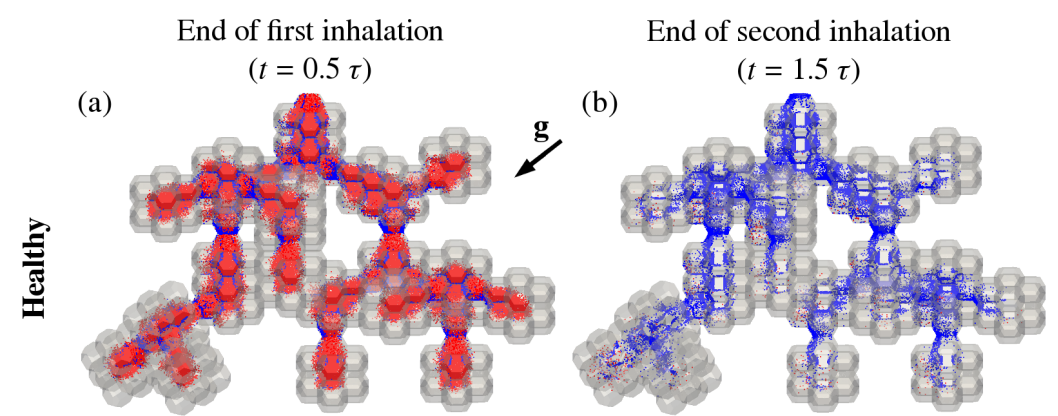

End of second exhalation
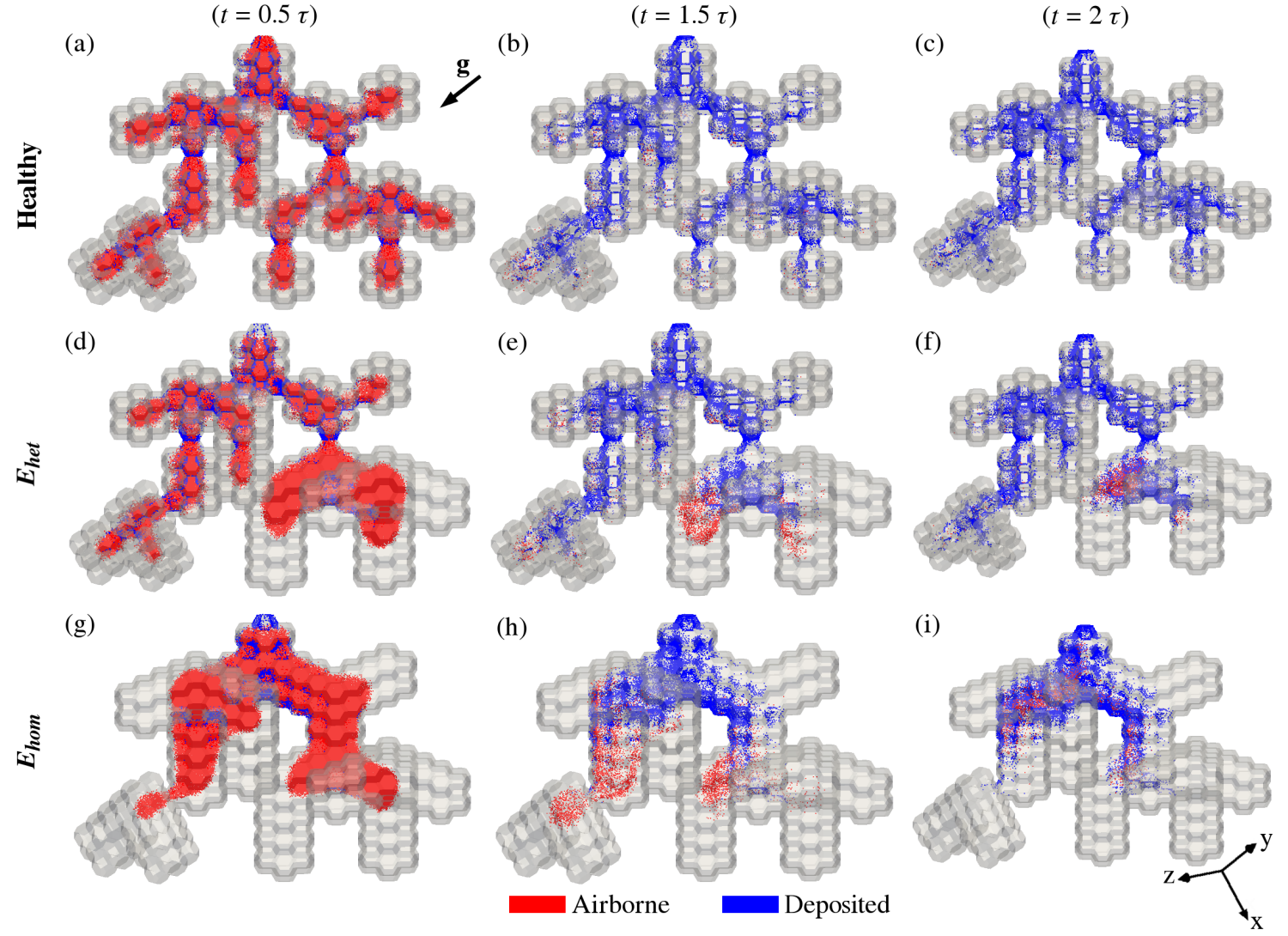

Figure 4: Snapshots of particle locations in the three acinar models captured at three time points during the breathing cycle. Particles in red are airborne (i.e. not deposited) and particles in blue are deposited. Corresponding movies of particle motions for the different cases are provided in the supplementary material (SM). 


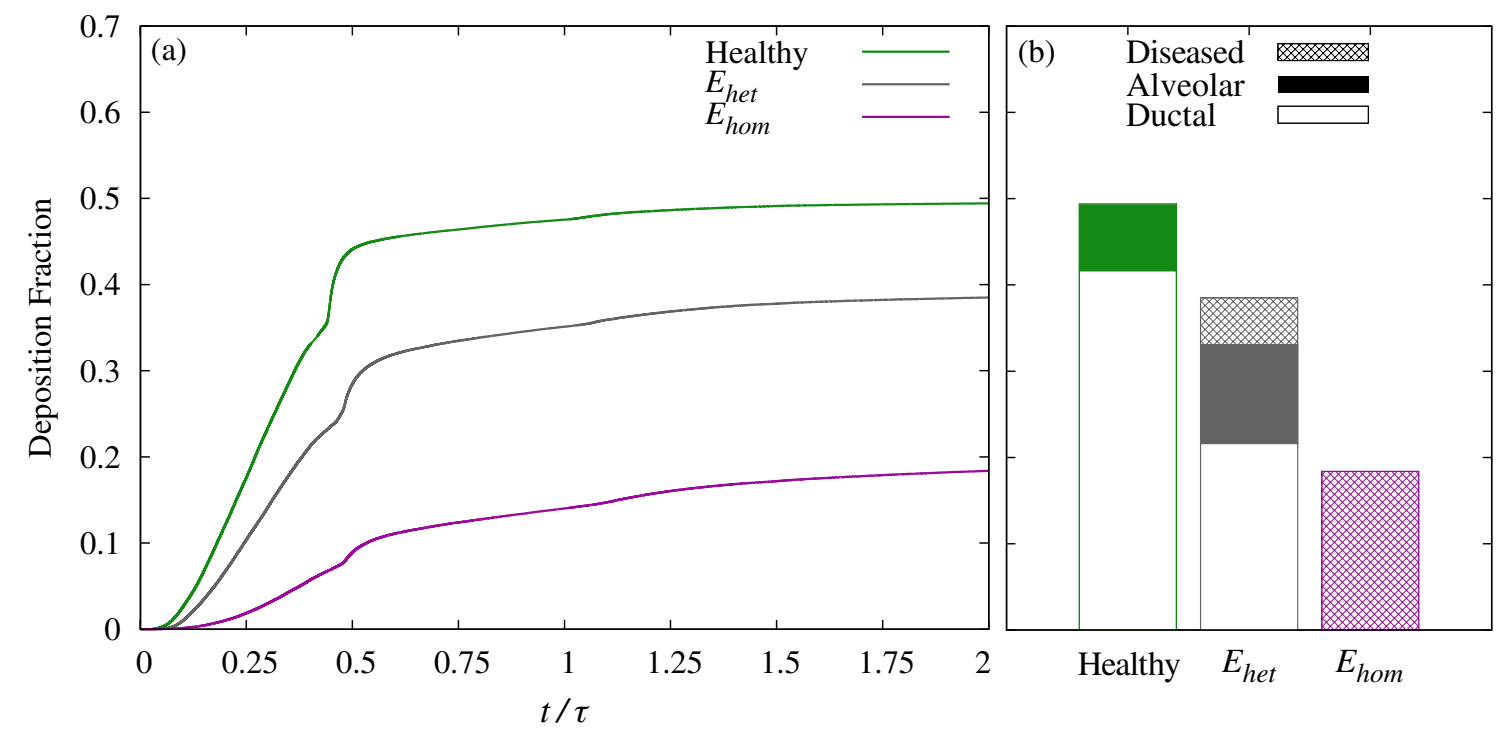

Figure 5: (a) Deposition fraction as a function of cumulative breathing cycles. Particles were injected throughout the inspiration phase of the first breath, from $t=0 \tau$ to $t=0.5 \tau$. (b) Final deposition fraction partitioned according to alveolar and ductal regions; the corresponding deposition fraction in the region of the emphysematous models is shown. Note that for such cases, the diseased regions cannot be distinguished according to alveolar and ductal regions in the absence of alveolar walls. 


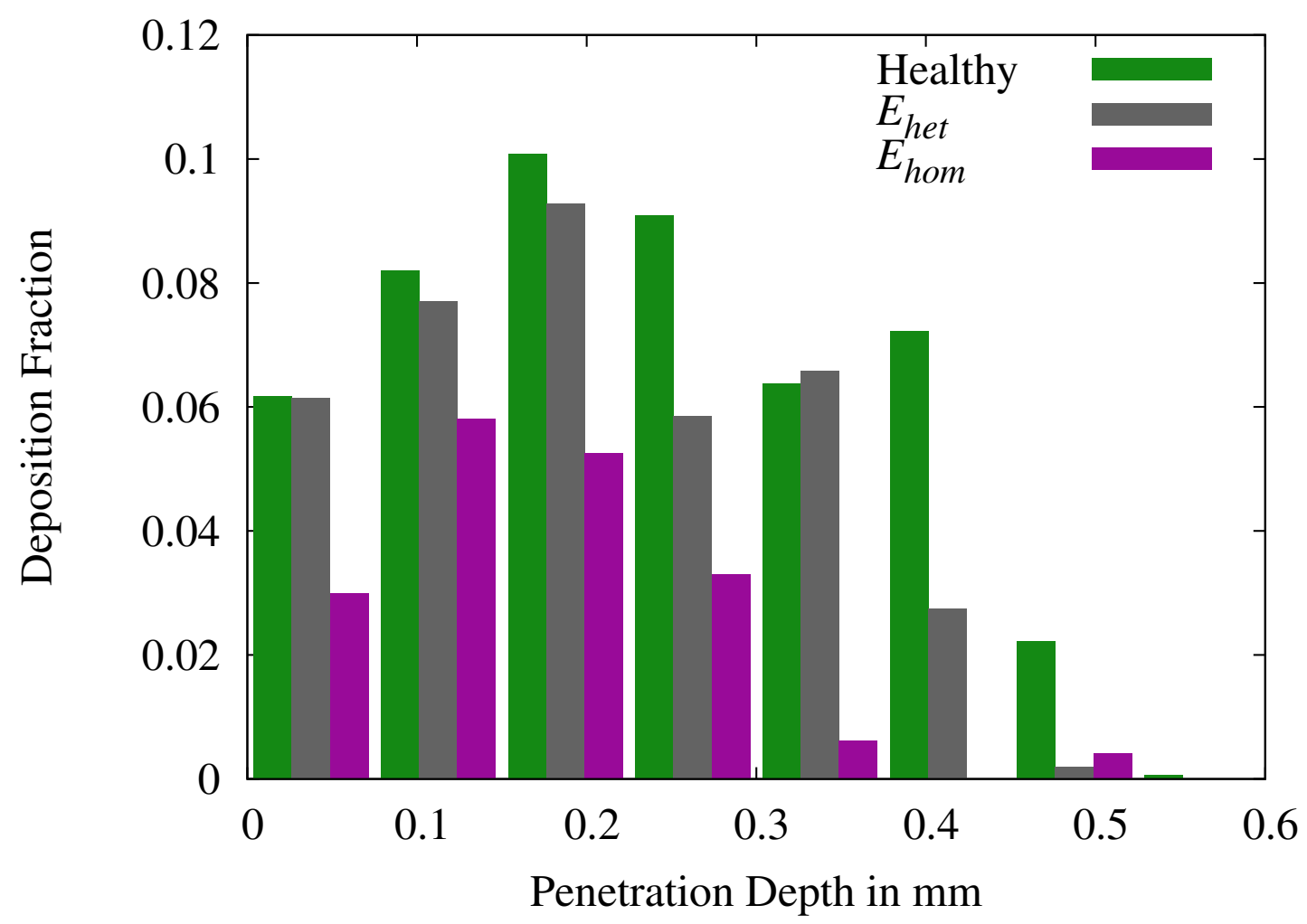

Figure 6: Deposition fraction plotted as a function of the penetration depth measured from the acinar entrance for the three acinar models, respectively. Penetration depths were taken after the second breath for deposited particles only, thus remaining airborne particles were not included in the calculation. Note that the penetration depths only assess the distance to the inlet of the domain, by calculating a particle's distance to the centreline of the duct and subsequently the distance (following the centreline of the duct) to the inlet. 
(a)

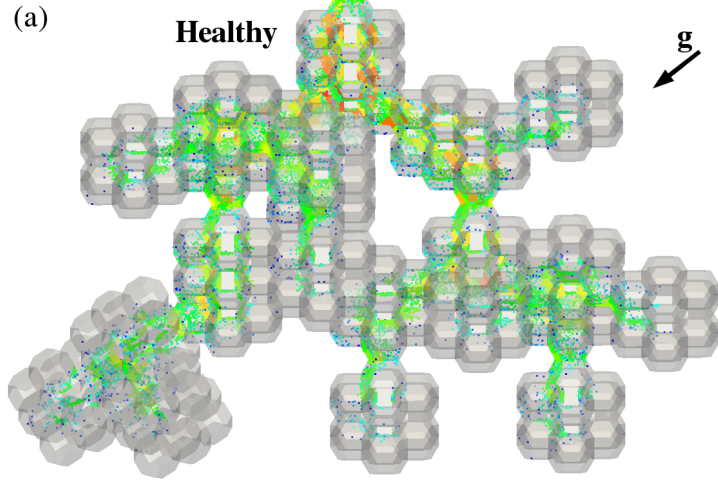

(c)

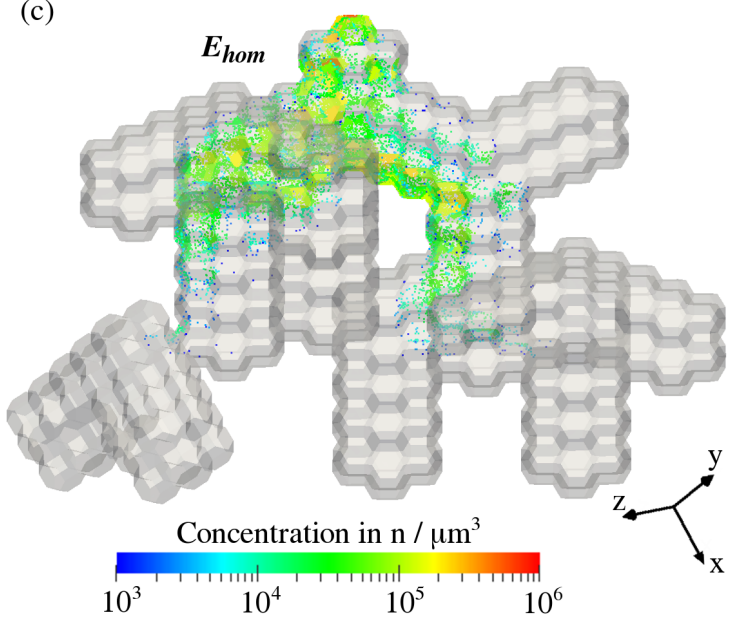

(b)

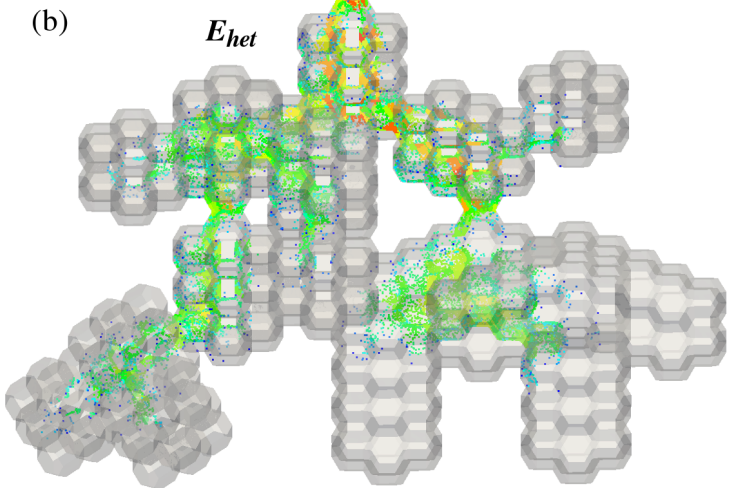

(d)

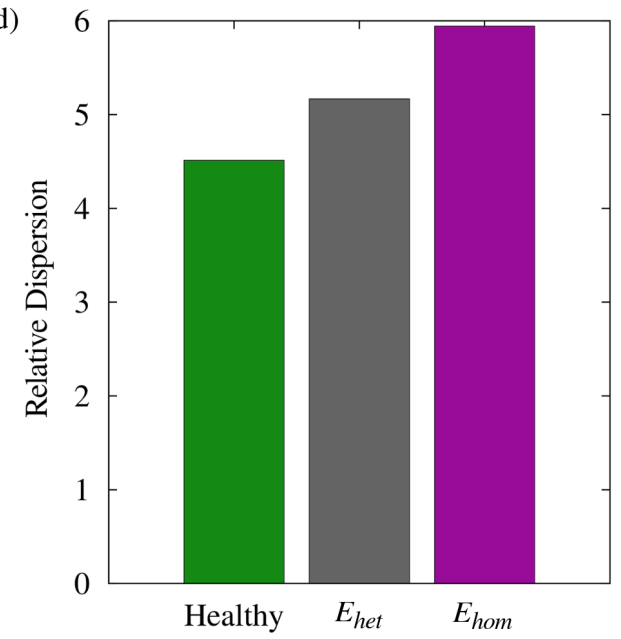

Figure 7: Deposited particle concentrations for the: (a) $H$, (b) $E_{\text {het }}$ and (c) $E_{\text {hom }}$ acinar models, respectively. Here, concentration is defined as the number of particles deposited within a voxel size with length of $10 \mu \mathrm{m}$. Panel (d) shows the relative dispersion $(R D)$, defined as the standard deviation normalized by the mean of the particle concentration for each acinar model [17]. Note that areas where particles did not deposit on were included in the $R D$ calculation. 


\section{List of Tables}

487 a Morphological properties of the healthy $(H)$, heterogeneous emphysematous $\left(E_{H e t}\right)$

488 and homogeneous emphysematous $\left(E_{H o m}\right)$ model at functional residual capacity

489 $(F R C)$. 31 
Table 1: Morphological properties of the healthy $(H)$, heterogeneous emphysematous $\left(E_{H e t}\right)$ and homogeneous emphysematous $\left(E_{H o m}\right)$ model at functional residual capacity $(F R C)$.

\begin{tabular}{lcccc}
\hline Model & Alveolar cavities & $\begin{array}{c}\text { Volume } \\
10^{-3} \mathrm{~mm}^{3}\end{array}$ & $\begin{array}{c}S / V \\
\mathrm{~mm}^{-1}\end{array}$ & $\begin{array}{c}\text { Tidal Volume } \\
10^{-3} \mathrm{~mm}^{3}\end{array}$ \\
\hline \hline Healthy, $H$ & 277 & 5.9 & 163.7 & 2.72 \\
Homogeneous Emphysema, $E_{\text {hom }}$ & 0 & 7.8 & 72.2 & 2.72 \\
Heterogeneous Emphysema, $E_{\text {het }}$ & 180 & 6.6 & 125.6 & 2.72 \\
$E_{\text {het }}:$ Normal Zone & 180 & 3.8 & 109.1 & 1.30 \\
$E_{\text {het }}:$ Diseased Zone & 0 & 2.8 & 73.9 & 1.42 \\
\hline
\end{tabular}

